\title{
Zur Rolle des Aufbau Verlags bei der Veröffentlichung des Werks des portugiesischen Romanciers Eça de Queirós in deutscher Sprache
}

\author{
https://dx.doi.org/10.12795/futhark.2014.i9.08 \\ Anette Kind \\ Universidade do Porto \\ akind@letras.up.pt
}

\begin{abstract}
This article discusses the impact of political circumstances on the translator's work. Especially under dictatorial regimes, the translator has to work with limited access to indispensable research tools. This will be demonstrated through the example of the translations into German of the literary works of the Portuguese writer Eça de Queiros, published by the Aufbau Verlag in East Berlin. The aim is to show how the publisher managed to reconcile an ambitious literary programme for the publication of world literature with the political and ideological requirements of the GDR cultural programme, and to describe the very difficult circumstances in which many translators had to work.

The existing documents in the Aufbau Verlag's archive - such as correspondence between the publishing house staff and the translators or cultural policy plans, reveal the desire by Aufbau Verlag to publish literary works carefully translated from a philological point of view but also strongly oriented towards social criticism, ideally towards Marxist ideas. The documents also show that, besides translator-specific problems like low fees and permanent time pressure, the translators had to work under regime-specific barriers such as material and logistics limitations or aggravating circumstances such as the refusal by the competent authorities
\end{abstract}


to issue travel permits to coss the inner-German border to carry out resanh in West Berlin's archives and libraries. It will also discuss the lack of travel opportunities to Portugal to obtain the philological works need. ed for the translations as well as to consult with local specialists to under. stand particularly complex source texts.

Key words: Literary translation, Eça de Queirós, Aufbau Verlag.

Abstract: Dieser Beitrag beschaftigt sich mit der Frage, welche Auswirkungen politische Unstände auf die Tätigkeit des Übersetzers haben konnen wenn dieser etwa in diktatorischen Regimes durch eingeschränkte Forschungs- und Bewegungstreiheit und somit ohne oder mit stark eingeschränktem Zugang zu den für ihn unentbehrlichen Hilsmitteln arbeiten muss(te). Dies soll am Beispiel der Übersetzungen der Werke des portugiesischen Autors Eça de Queirós in die deutsche Sprache aufgezeigt werden, die vom Aufbau-Verlag herausgegeben wurden. Dabei soll der Frage nachgegangen werden, inwiefern einerseits dem Verlag die Gratwanderung gelungen ist, ein anspruchsvolles literarisches Verlagsprogramm zur Veröffentlichung von Werken der Weltliteratur mit den politisch-ideologischen Vorgaben des DDRKulturprogramms zu vereinbaren, und andererseits, unter welchen, ihre Recherche-Arbeit erschwerenden Umständen die Übersetzer oft gearbeitet haben.

Die im Archiv des Aufbau-Verlags vorhandenen Unterlagen, etwa Briefwechsel zwischen Lektoratsmitarbeitern und Übersetzern oder periodisch angefertigte kulturpolitische Themenpläne des Verlags, dokumentieren den Anspruch des Verlags, unter philologischen Gesichtspunkten sorgfältig angefertigte Übersetzungen von Werken mit einem im Idealfall manistischen, zumindest jedoch sozialkritischen Ansatz zu publizieren. Sie belegen neben den für die Tätigkeit des literarischen Übersetzers typischen Umständen wie permanenten Zeitdruck oder mangelnde Vergütung aber auch regime-spezifische Hindernisse wie materiell-logistische Einschränkungen, vor allem aber die Klagen über darüber hinaus erschwerende Umstände, etwa die Weigerung der zuständigen Behörden, einen Passierschein zur Recherche in West-Berliner Archiven und Bibliotheken auszustellen, oder die mangelnden Verbindungen oder Reisemöglichkeiten nach Portugal, zwecks Beschaffung der zur Übersetzung notwendigen philologischen Werkausgaben oder der für das Verständnis besonders komplexer Ausgangstexte notwendige Rücksprache mit Spezialisten vor Ort. 
Schlagwörter: Úbersetzung, Literarische Übersetzung, Eça de Queirós, Aufbau Verlag.

Die erste Publikation eines Textes von Eça in deutscher Sprache (Stadt und Gebirg, ubersetzt von Luise Ey) ist auf das Jahr 1903 datiert. In der ersten Hălfte des 20. Jahrhunderts erschienen in deutscher Sprache desweiteren die Romane Vetter Basilio und Das Verbrechen des Pater Amaro sowie einige Erzählungen. Der eigentliche Boom der EçaRezeption in Deutschland geht jedoch auf die Initiative des Ostberliner Aufbau Verlags zurück, der fast ausschließlich für die Publikationen der 50-er und 60-er Jahre verantwortlich ist, sowie für viele Neuauflagen dieser Werke in den folgenden Jahrzehnten. Ungefähr die Hälfte aller ins Deutsche übersetzten Texte von Eça de Queirós sowie ihre Neuauflagen erschienen in diesem Verlag. Angesichts der Tatsache, dass der Aufbau Verlag erst nach Ende des 2. Weltkriegs gegründet wurde, ist dies ein beachtlicher Beitrag zur Eça-Rezeption in Deutschland.

Fast durchgängig wurden die Übersetzungen dieser Texte verlagsintern angefertigt, und zwar bis auf eine Ausnahme ${ }^{1}$ von den Übersetzern Willibald Schönfelder und Rudolf Krügel. Bei der Publikation des Werks von Eça de Queirós legte der Aufbau Verlag seinen Schwerpunkt auf die großen Gesellschaftsromane. So wurden von den acht eindeutig als Roman einzuordnenden Prosatexten Eças sechs übersetzt und veröffentlicht. Im Verlagsprogramm erscheinen auch kürzere Prosatexte wie Der Mandarin oder José Matias, stellen aber eher eine Randerscheinung dar. Ganz anders dagegen veröffentlichten im übrigen die westdeutschen Verlage von Eça nur wenige Romane und einige kürzere Prosatexte, später dann auch einige Romane als Lizenzausgaben des Aufbau Verlags.

Das Archiv des Aufbau Verlags wird heute in der Berliner Staatsbibliothek als Depositum verwaltet und ist der Forschung in

\footnotetext{
${ }^{1}$ Es handelt sich um die Übersetzung des Romans Die Reliquie, die der Aufbau Verlag ab 1951 in der ursprünglich im Kurt Wolff verlegten Übertragung von Richard A. Bermann aus dem Jahre 1918 herausgab; 1984 wurde diese, voraussichtlich aufgrund einer juristisch ungeklärten Vertragslage bezüglich der Bermann'schen Fassung, durch die Übersetzung von Andreas Klotsch abgelöst, der diese als externer Mitarbeiter angefertigt hat.
} 
digitalisierter Form zugănglich. Aus den dort einsehbaren Shriftstücken geht anschaulich und in der Regel gut dokumentiert hervor, wie der Verlag bei der Auswahl der zu publizierenden Werke vorgegangen ist, aber auch, sofem es dokumentiert ist, wie sich die Arbeit der Übersetzer konkret gestaltete und mit welchen Schwierigkeiten sie konfrontiert waren.

Um deutlich zu machen, unter welchen Vorzeichen die Auswahl der Werke Ecas vorgenommen wurde, sei zunächst ein kurzer therblick über die Geschichte des Verlags und seine programmatische Zielsetzung gegeben. Im folgenden soll dann exemplarisch verdeutlicht werden, wie der komplizierte Vorgang von der Vorauswahl eines Werkes über verlagsinterne Verfahren bis hin zur Druckgenehmigung ablief. Im Anschluss daran soll am Beispiel des Übersetzers Krügel, dessen aufschlussreiche Korrespondenz mit dem Verlag sehr gut dokumentiert ist, aufgezeigt werden, welche Hürden sich dem Übersetzer bei seiner Tätigkeit in den Weg stellten.

Gegründet wurde der Aufbau Verlag 1945 in der sowjetischen Besatzungszone und entwickelte sich dann schnell zum führenden Verlag für Belletristik in der DDR. ${ }^{2}$ Von seiner Gründung an veröffentlichte der Verlag Klassiker der deutschen wie der ausländischen Literatur, zu Beginn auch schwerpunktmäßig Werke von Autoren, die unter den Nazis ins Exil geflüchtet waren, wie auch explizit antifaschistische Literatur. Ein weiterer Schwerpunkt im Laufe der folgenden Jahrzehnte war die Publikation zeitgenössischer Literatur der DDR wie des Auslands, wobei der Literatur aus den osteuropäischen sozialistischen Staaten und aus Lateinamerika ein besonderes Gewicht zukam. Wie das gesamte kulturelle Geschehen in der DDR wurde auch der Aufbau Verlag durch die von der SED eingesetzten Zensurorgane kontrolliert, da die Literatur von der Kulturpolitik als Mittel im Klassenkampf instrumentalisiert wurde. Dennoch gelangen dem Verlag immer wieder Publikationen westlicher, auch vieler westdeutscher Autoren. Der Kampf für mehr publizistische wie politische Freiheit führte Ende der 50-er Jahre zu

\footnotetext{
${ }^{2}$ Genaueres zur Verlagsgeschichte $2 . B$. unter:

http://staatsbibliothek-berlinde/suche/einzelansicht/article/2006-11-28-4034/ (Zugriff 25.1.13).
} 
Schauprozessen, in denen Verlagsleiter Walter Janka und andere etwas zu forsch fortschreitende Mitarbeiter der Verlagsleitung zu Haftstrafen verurteilt wurden. Daraufhin wurden wieder verstärkt Titel mit explizit marxistischem Gedankengut ins Verlagsprogramm aufgenommen. Trotz des permanenten Eingriffs der Zensurbehörden gelang dem Verlag bis zur Auflösung der DDR durch taktisch geschicktes, auch mutiges Vorgehen der jeweiligen Lektorats- und Verlagsleiter immer wieder die Publikation kritischer Texte und anspruchsvoller Literatur, die eigentlich den strengen ideologischen Vorgaben nicht genügen konnte. Voraussetzung für eine Aufnahme ins Verlagsprogramm war freilich neben literarischer Qualität auch immer ein sozial oder politisch relevanter Ansatz.

Die programmatische Zielsetzung des Aufbau Verlags wurde jährlich in sogenannten Themenplänen festgelegt. So heißt es in einem Verlagsprotokoll aus dem Jahre 1969 beispielsweise: „Unser Bestreben [...] war es, im Rahmen der uns zur Verfügung stehenden Kapazität unseren vielfältigen Aufgaben nachzukommen: das kulturelle Erbe der Weltliteratur durch die Herausgabe von Fortsetzungsbänden großer Werkausgaben, Einzelbänden und Bänden der $\mathrm{BDW}^{3} \mathrm{zu}$ pflegen, den Leser der DDR mit den progressiven bürgerlichhumanistischen Traditionen der ausländischen Literaturen bekannt zu machen, ihm bedeutende Werke älterer sozialistischer Autoren vorzustellen und ihm einen Einblick in die moderne Literatur des sozialistischen und kaptitalistischen Auslands zu geben. Dabei waren wir bemüht, möglichst viele Literaturen einzubeziehen, um auch auf diese Weise eine thematische Breite und Vielfalt zu erzielen." 4

Die Verlagsleitung, eingezwängt in ein Korsett politischer und ideologischer Vorgaben, war also einerseits bemüht, den parteipolitischen Vorstellungen der SED zu genügen, vertrat andererseits aber auch den Anspruch, literarisch wertvolle Bücher verlegen.

Es gehörte zur Verlagspraxis, in sogenannten Titelannahmeverfahren darüber zu befinden, ob der Verlag sich um

${ }^{3}$ Abkürzung für Bibliothek der Weltliteratur; Bezeichnung einer Reihe des Verlags.

${ }_{4}^{4}$ Archiv Aufbau-Verlag, SSB IIIADep38 E0054 0044 r [44 und 45 von 247]. 
die Publikation eines bestimmten Werks bemühen sollte. Im Rahmen dieser Titelannahmeverfahren wurden zwecks Vorauswahl sogenannte Innengutachten durch Mitarbeiter des Verlags oder Außengutachten durch exteme Mitarbeiter in Auftrag gegeben. In diesen Gutachten sollte das Werk ideologisch und auf literarische Relevanz hin analysiert werden. Wenn sich die Verlagsleitung aufgrund positiver Gutachten für eine Publikation entschied, wurde beim Ministerium für Kultur ein Druckgenehmigungsantrag eingereicht, dem gegebenfalls die erwähnten Gutachten beigefügt wurden. $1 \mathrm{~m}$ Falle von ausländischen Werken wurden diese i.d.R. erst nach erfolgter Druckgenehmigung übersetzt. Die Druckgenehmigungsanträge nun spielten eine entscheidende Rolle in einem Staat, in dem der gesamte Literaturbetrieb überwacht wurde und die literarischen Werke der Zensur unterworfen waren. Da diese Texte als Dokumente zwischen Verlagen und Ministerium für Kultur zu verstehen sind, können sie auch als mehr oder minder kaschierte Umgehung der Zensur im Rahmen eines Druckgenehmigungsverfahrens zu sehen sein und sind somit unter Vorbehalt zu lesen. Sie waren dazu bestimmt, ein als literarisch wertvoll und inhaltlich relevant beurteiltes Werk vor den staatlichen restriktiven Entscheidungsträgern aus sozialistischer Sicht als ideologisch vertretbar darzustellen. In diesen Anträgen sind daher auch keine kritischen Anmerkungen zu den Werken verzeichnet, wohl aber in den verlagsintern entstandenen Dokumenten im Rahmen des Titelannahmeverfahrens. ${ }^{5}$

Wie gestalten sich nun diese Vorgänge hinsichtlich der Publikation der Texte von Eça de Queirós, über dessen Werk im Auslandslektorat befunden wurde? Soweit es aus den Archivunterlagen hervorgeht, griff Lektoratsleiter Günter Caspar ${ }^{6}$ u. a. auf Gutachten des Übersetzers und Schriftstellers Anton Antkowiak und des Übersetzers Rudolf Krügel zurück. Beide haben sich intensiv mit dem Werk von Eça auseinandergesetzt, wenn auch aus unterschiedlichen Perspektiven: während ersterer sich in seinen Ausführungen

${ }^{5} \mathrm{~S}$. dazu: KIRSTEN, Jens, Lateinamerikanische Literatur in der DDR. Publikations- und Wirkungsgeschichte, Berlin, Christoph Links Verlag, 2004, S. 105-106.

'Ab 1955 Lektor, ab 1956 Cheflektor , ab 1963 Leiter des Lektorats "Zeitgenössische deutsche Literatur" beim Aufbau Verlag. 
vorrangig auf kritische Literatur über den Autor stützt, hat sich zweiterer zwar ebenfalls in die Kontexte und historischen Hintergrunde der Werke sorgfältig eingearbeitet, ist aber durch die intensive Auseinandersetzung mit den Texten beim Übersetzen und durch gründliche Lektüre der einschlägigen Sekundärliteratur mit den stilistischen Merkmalen bestens vertraut und kann die Texte somit auch in ihrer literarischen Qualität beurteilen. Antkowiak und Krügel kommen in ihren Gutachten oft zu unterschiedlichen Urteilen: Antkowiak erweist sich als den ideologischen Vorgaben enger verhaftet und hält nur die großen Gesellschaftsromane im Rahmen einer Werkauswahl für publikationswert, da diese die sozialen Widersprüche der portugiesischen Gesellschaft des 19. Jahrhunderts aufdecken und offenkundig verurteilen. Die Kurzprosa dagegen, übrigens ebenso wie Eças non-fiktionale Texte, also seine Essays und politischen Schriften, verurteilt er in Bausch und Bogen als minderwertig, bezeichnet sie abfällig als "Randerscheinungen” oder „Vorübungen zu seinen Romanen”, deren „künstlerische Bedeutung "gleich Null" sei und die somit nicht der Veröffentlichung wert seien („Eça war unfähig, die kleine Form zu bewältigen”). Krügel dagegen legt am 18.7.55 ein mehrseitiges Schreiben vor, in dem er eine Auswahl aus den "Contos" empfiehlt. Diese, so begründet er seine Einschätzung,

knüpfen praktisch an den realistischen Endpunkt der "Prosas bárbaras" an und führen über die noch recht zurückhaltende soziale Kritik (z.B. in "José Matias") zu humanistischen Gedankengängen... ${ }^{8}$

Man kann also festhalten, dass sich Antkowiak als der linientreue Gutachter erweist, der diejenigen Werke Eças zur Veröffentlichung empfiehlt, die ihn als gesellschaftskritischen, an sozialistischen Vorbildern geschulten Autor ausweisen, während Krügel neben inhaltlichen verstärkt philologische und literarische Aspekte geltend macht.

Der betont marxistische Jargon des Außengutachters Antkowiak (zeitweise auch Mitarbeiter des Aufbau Verlags, seit 1953 Leiter des

${ }^{7}$ Archiv Aufbau Verlag, SBB IIIA Dep38 A0197a 0096 r.

${ }^{8}$ Archiv Aufbau Verlag, SSB IIIADep38 A0197a 0098 r [99 von 230]. 
Lektorats für Realismus des 19. Jahrhunderts), erhellt sich übrigens in einem anderen Licht, wenn man die Tatsache bedenkt, dass nach dem Zusammenbruch der DDR durch Einsicht in die Stasi-Akten ersihtlich wurde, dass er vom Ministerium für Staatssicherheit gezielt im Verlagswesen, in dem er sich auskannte, als IM (Inoffizieller Mitarbeiter) eingesetzt wurde, der seine Kollegen bespitzelte und regelmäßig Berichte über Arbeitsweisen und Verlagspraktiken des Aufbau Verlags vorlegte. Er bespitzelte seine Kollegen und legte regelmäßig Berichte über Arbeitsweisen und Verlagspraktiken des Aufbau Verlags wie auch des Verlags Volk und Welt vor, in denen er beispielsweise die liberalen und eher literarisch als strikt ideologisch orientierten Auswahlkriterien der Lektoratsleiter anprangerte. 9 Auf die Auswahl der für die Veröffentlichung vorgesehenen Bücher übte er auch Einfluss aus und verhinderte die Publikation literarisch anspruchsvoller Werke, sowohl im Verlag Volk und Welt (zunächst als Lektor und nach seinem Ausscheiden aus dem Verlag ab 1961 als Gutachter ${ }^{10}$ ) wie auch im Aufbau Verlag, indem er negative, von einer Publikation abratende Gutachten vorlegte, wenn er die betreffenden Werke als nicht explizit marxistisch und somit aus ideologischer Sicht nicht für vertretbar hielt. Viele Werke stempelte er als bürgerlich dekadent $a b$, schreckte in seinen Beurteilungen sowohl der Werke wie der envolvierten Verlagsmitarbeiter auch vor äußerst subjektiv-polemischen Bezeichnungen wie "völlig unbrauchbar, dekadent, widerlich fast" nicht zurück. Aus einer Stasi-internen Einschätzung des IM Antkowiak geht hervor, dass er als übereifriger, oft über die ihm gesteckten Ziele hinausschießender Zensor

9 Ursprünglich selber aufgrund staatsgefährdender Propaganda zu einer Zuchthausstrafe verurteilt, wurde er dort als IM gewonnen. In der DDR wie in anderen kommunistischen diktatorischen Staaten war die Selbstkritik gängige Praxis. So hatte sich Antkowiak während der Haft selbst bezichtigt, "da $\beta$ die DDR zeitweise mit westlicher dekadenter Literatur überschwemmt wurde". (Vgl. BARCK, Simone, Antifa-Geschichte(n). Eine literarische Spurensuche in der DDR der 1950er und 1960er Jahre. Köln: Böhlau Verlag, 2003, S. 110.) Seine spätere Spitzeltätigkeit in diversen Verlagen stellte er in den Dienst der Bekämpfung westlicher Literatur im Besonderen und der ausländischen Literatur im Allgemeinen, der er generell misstrauisch gegenüberstand, da er in ihr einen gefährlichen Wortführer zur Verbreitung dekadenten Gedankenguts sah.

${ }^{10} \mathrm{vgl}$. KIRSTEN, S. 125 
einzuschätzen war." Wohl aus diesem Gund gelang es ihm auch nicht, wie Kirsten feststellt, die Verlagspolitik des Verlags Volk und Welt oder des Aufbau Verlags wesentlich zu beeinflussen, wenn er auch die Publikation einiger Werke erfolgreich verhinderte.

Darüber, wie sich die Arbeit des Übersetzers vor dem Hintergrund der Verlagspolitik darstellt, geht wenig aus den Archivunterlagen hervor. Es finden sich zwar Abrechnungsbelege und Übersetzungsaufträge, aber nur wenige Briefwechsel zwischen Übersetzern und Verlagsmitarbeitern. Eine Ausnahme bildet der Übersetzer Rudolf Krügel, von dem zahlreiche Briefe sowie weitere Verlagsunterlagen vorhanden sind, in denen er namentlich Erwähnung findet.

Den Großteil der Werke von Eça, insbesondere die Gesellschaftsromane, wurden von Krügel übersetzt. Seine produktive, aber auch problematische Zusammenarbeit mit dem Verlag ist besonders gut dokumentiert, da er einen intensiven Briefwechsel mit den Verlagsmitarbeitern führte. Dieser Umstand ist vor allem der Tatsache geschuldet, dass er in seinen mit den Übersetzungen verbundenen Nachforschungen besonders gewissenhaft und akribisch vorgegangen ist, daher wiederholt die Fristen nicht einhalten konnte bzw. das Honorar dann in keinem Verhältnis mehr zum Zeitaufwand stand und er in diesen Angelegenheiten diverse Bittbriefe an den Verlag richtete. $\mathrm{Zu}$ seiner extremen Gewissenhaftigkeit beim Übersetzen gesellt sich eine permanente und nur mühsam kaschierte Ärgernis über das sozialistische System mit all seinen Beschränkungen, die nicht nur dem normalen Bürger das Leben erschwerten, sondern ganz besonders dem Übersetzer, dem der Zugang zu vielen für die Arbeit notwendigen Quellen und Hilfsmitteln verwehrt blieb.

Die Briefe von Krügel dokumentieren auf aufschlussreiche Weise nicht nur die Arbeitsweise dieses Übersetzers im Besonderen, sondern generell die Bedingungen, unter denen die Übersetzer in der DDR und somit auch im Aufbau Verlag gearbeitet haben. Ein Übersetzer, der komplexe und stilistisch ausgefeilte Texte wie die von Eça

${ }^{11}$ Ebenda, S. 126 
übersetzt, Texte voller Anspielungen und Hinweise, die oft nur dem gebildeten portugiesischen Leser vollständig verständlich sind, ist auf eine intensive Recherche angewiesen, um sich den Text in all seinen Nuancen zu erschließen. Diese Nachforschungen erfordern die Konsultation von Lexika und Wörterbüchern, die Lektüre literaturwissenschaftlicher Arbeiten von Experten der Ausgangskultur, eventuell das Hinzuziehen von mit Anmerkungen versehenen Ausgaben in der Ausgangssprache. Sie erfordern aber im Falle besonders diffiziler Texte auch eine Beratung durch Experten der Ausgangskultur, um nicht nur die lexikalische Dimension, sondern auch alle mehr oder minder kodifizierten Hinweise des Originals vollständig $\mathrm{zu}$ erfassen, was beispielsweise bei historisierenden Texten von Bedeutung ist.

Dass Krügel extrem sorgfältig gearbeitet hat, ist in seinen Briefen an den Verlag eindrücklich dokumentiert. Wiederholt macht er darauf aufmerksam, dass sein Bestreben, "das Letzte aus dem Original herauszuholen"12 mit einem nicht zu unterschätzenden Zeitaufwand verbunden ist.

So begnügt er sich keineswegs damit, die vom Verlag in Auftrag gegebenen Übersetzungen postalisch einzureichen, sondern diese werden stets von mehrseitigen engzeilig mit Schreibmaschine verfassten Begleitschreiben an den zuständigen Lektoratsleiter geschickt. In seinen Erläuterungen legt Krügel meist eine ausführliche Stilanalyse vor und weist auf die Besonderheiten des betreffenden Textes im Rahmen des Gesamtwerkes von Eça hin. Die teils in pedantischer Ausführlichkeit dokumentierten stilistischen Merkmale des betreffenden Textes erfüllen verschiedene Funktionen. So scheint es ihm auf der einen Seite ein persönliches Anliegen zu sein, die während der intensiven Auseinandersetzung mit dem Text gewonnenen Einsichten mitzuteilen. Bei stilistisch besonders komplexen Texten (so beim Grafen von Abranhos ${ }^{13}$ ) möchte er mit seinen Ausführungen aber auch dafür um Verständnis werben, dass er sich nicht in der Lage sieht, die Übersetzung innerhalb der vertraglich festgelegten Frist vorzulegen oder aber er bittet den

\footnotetext{
${ }^{12}$ Archiv Aufbau-Verlag, SBB IIIA Dep38 A0384 0091 r.

${ }^{13}$ Archiv Aufbau-Verlag, SBB IIIA Dep38 A0384 $0121 \mathrm{r}$.
} 
zuständigen Verlagsmitarbeiter, angesichts der Komplexität des zu ubersetzenden Textes und der sich daraus ergebenden außerordentlich zeitintensiven Recherchearbeit eine höhere Vergütung in Erwägung zu ziehen.

In den Vorverhandlungen zu den geplanten Übersetzungen von vier Werken Eças begründet er in einem Schreiben vom 11.7.1957 an den damaligen Cheflektor Günter Caspar seine Bitte um einen höheren Honorarsatz mit dem Hinweis auf die "weit überdurchschnittlich hohen literarisch-künstlerischen Anforderungen, die eben gerade der 'Graf von Abranhos' an den Übersetzer stellt"14, also an eins der zu übersetzenden Werke, und fährt fort: „Schließlich wird Ihnen auch jeder andere Übersetzer bestätigen, da $\beta$ es ein beträchtlicher Unterschied ist, ob man einen lediglich deskriptiven Unterhaltungsroman vor sich hat oder ein bewußt stilistisch affektiertes und noch dazu stark ironisierendes Werk."

In einem Schreiben vom 28.1.1960 ${ }^{15}$ weist Krügel auf die außerordentlichen Schwierigkeiten hin, die die Übersetzung des EçaTextes Das berühmte Haus Ramires bereitet: in die Erzählung ist eine Novelle aus dem 13. Jahrhundert eingefügt, die Vokabeln beinhaltet, die in keinem Wörterbuch $\mathrm{zu}$ finden sind. Auch postalische Nachfragen bei Portugiesen bleiben ergebnislos. Orts- und Eigennamen sind in mühevoller Kleinarbeit und mit Hilfe von Fachliteratur zu klären.

Als ihm nahegelegt wird, die Übersetzung, deren Frist er anscheinend nicht einhalten kann, aus der Hand zu geben, schreibt er in seiner Antwort:

Eça de Queiroz war selbst sprachschöpferisch tätig, wie man sogar in einem neueren hiesigen Lexikon (Band A-K 1956) auf S. 437 nachlesen kann. Was einem Übersetzer, der seine Aufgabe ernst nimmt, dabei blüht, geht sehr eindeutig aus einem Artikel von Zdeñ̌k Hampejs im Märzheft 1958 der "Deutschen Literaturzeitung”, Sp. 219-22 hervor,

${ }_{14}$ Archiv Aufbau-Verlag, SBB IIIA Dep38 A0384 0117 r.

${ }^{15}$ Archiv Aufbau-Verlag, 006465 von 267.

Futhark 9 (2014)

Kind, Zur Rolle des Aufbau Verlags, 221-236

ISSN 1886-9300 
der dort ein unlängst in Coimbra erschienenes Spezialwerk abber Eça de Queiroz's Sprache und Stil bespricht. ${ }^{16}$

Hier bezieht sich Krügel auf die 1954 erschienene Studie von Emesto Guerra da Cal Linguagem e Estilo de Eşa de Queirós, die auch heute noch als Standardwerk zu Eças Stil und literarischer Sprache gilt. ${ }^{17}$ Aus den Archivunterlagen lässt sich nicht zweifelsfrei entnehmen, ob Krügels Gesuchen auf Fristverlängerung bzw. ein höheres Honorar jeweils entsprochen wurde, jedoch wird zumindest in einigen Fällen der Antrag auf höhere Honorierung durch Verlagsmitarbeiter positiv beschieden. ${ }^{18}$ Zweifelsfrei belegen lässt sich jedenfalls die Tatsache, dass seine Qualitäten als gewissenhaft und genau vorgehender Übersetzer in diversen Verlagsnotizen und Berichten wiederholt Anerkennung fanden. So heißt es in einem Verlagsschreiben, Krügels Übersetzung der Maias sei „mit Sorgfalt und Überlegung angestellt worden” und könne als „zufriedenstellend angesehen werden" 19 .

Es war sicherlich ein Glücksfall für den Aufbau Verlag, einen geradezu besessen sorgfältig arbeitenden Übersetzer unter Vertrag zu haben, der seine Übersetzungen fast als Mission im Namen des portugiesischen Schriftstellers begreift. Dass er aber von der zeitaufwendigen Arbeit allein nicht satt wird, erklären die diversen mit ausführlichen Erklärungen versehenen Bittbriefe um Erhöhung des Honorars.

\footnotetext{
${ }^{16}$ Ebenda.

${ }^{17}$ CAL, Emesto Guerra, Linguagem e Estilo de Eşa de Queirós, Lissabon, Editorial Aster, 1954.

${ }^{18}$ Archiv Aufbau-Verlag, SSB IIIA Dep38 A0384 0033 [00 von 267]: Ein Kommentar vom 27.2. 1961 zu Krügels Übersetzung von Das berühmte Haus des Ramires: "Das Ergebnis der Stichproben, die ich Rudolf Krügels Übesetzung (sic) machte, war recht befriedigend. Die Übersetzung ist mit Sorgfalt und Überlegung angefertigt. In Hinblick auf den Schwierigkeitsgrad des Textes (komplizierter Satzbau, ironische Färbung vieler Passagen, eingestreute Verse, Anspielungen auf historische Ereignisse, sehr reiches Vokabular) erscheint mir eine Honorierung von $15 \mathrm{DM}$ je Seite angemessen." Unterzeichnet von Elga Abramowitz (Übersetzerin beim Aufbau-Verlag).

${ }^{19}$ Archiv Aufbau-Verlag, SBB IIIA Dep38 A0384 0002.
} 
Ein weiteres Problem, mit dem sich Krügel konfrontiert sieht und das ihn bei den für eine philologisch sorgfältige Übersetzung notwendigen Recherchetätigkeiten einschränkt, besteht in der Tatsache, dass er seit Mauerbau die Westberliner Bibliotheken nicht mehr aufsuchen kann. So bittet Krügel am 1.9.1961 (also knapp drei Wochen nach Mauerbau) um einen Dauerpassierschein für den Besuch der Ibero-Amerikanischen Bibliothek in Berlin-Lankwitz, um sich über Neuerscheinungen und den Forschungsstand sowohl auf seinem Fachgebiet als Übersetzer als auch im Rahmen seiner Lehrtätigkeit als Lektor für Portugiesisch (an der Hochschule für Ökonomie Berlin Karlhorst) zu informieren. Dieser Passierschein wurde ihm anscheinend nicht bewilligt, denn er weist in einem Schreiben vom 28.12.62, also ein gutes Jahr später, auf die mit der Übersetzung von Eça verbundenen Schwierigkeiten hin, die es erforderlich machen, Spezialwörterbücher, ältere Enzyklopädien etc. $\mathrm{zu}$ konsultieren, und betont in diesem Zusammenhang erneut die Notwendigkeit der Ausstellung eines Passierscheins. Im Gegensatz zu anderen Mitarbeitern des Aufbau Verlags habe man ihm diesen nicht ausgestellt, sondern ihn immer wieder vertröstet.

„Die Übernahme weiterer Übersetzungen aus meinem Fachgebiet - insbesondere solcher schwieriger älterer Autoren - „so fährt er fort, hätte für mich dann auch nur Sinn, wenn ich erforderlichenfalls die in beiden Teilen Berlins vorhandenen Konsultationsmöglichkeiten nutzen kann. Irgendwelche Nachlässigkeiten auf Kosten der Qualität werde ich mir jedenfalls nicht leisten, denn gewisse Kreise in Westdeutschland warten nur darauf und möchten daraus gern politisches Kapital schlagen (vgl. DIE WELT vom 8.10.1960 über Vetter Basilio")."20

Dieses permanente Ringen um die bestmögliche Übersetzung unter Bedingungen, die eine angemessene Recherche nicht eben erleichtern, nahm zum Teil dramatische Dimensionen an. Der permanente Zeitdruck bei gleichzeitig ebenso konstanter Geldknappheit, da er die Arbeiten meist nicht termingerecht einreichen konnte, führten zu einer so großen nervlichen Belastung,

20 Archiv Aufbau-Verlag, SSB IIIADep38 A0197a 0125 r [128 von 230]; SSB IIIADep38 A0384 0024 [24 von 267]. 
dass er anscheinend in regelmäßigen Abständen in eine Nervenheilanstalt eingewiesen werden musste. Caspar schreibt an die Verlagsleitung, das Lektorat „möchte ilm aber trotzdem nicht als Mitarbeiter verlieren, weil seine Arbeit in seinen normalen Zeiten für uns von unschätzbarem Wert ist". ${ }^{21}$

Hinzu kommt, dass Krügel dem sozialistischen Regime sicherlich nicht unkritisch gegenüberstand, dies jedoch aus verständlichen Gründen nur sehr vermittelt zum Ausdruck bringen konnte. Seinem Leiden an der restriktiven DDR-Politik, die u.a. Reisen in den Westen unmöglich machte und damit nicht nur die persönliche Freiheit ihrer Bürger empfindlich einschränkte, sondern eben auch die Recherchemöglichkeiten des Übersetzers, verlieh er wiederholt in Zitaten anerkannter Parteigenossen Ausdruck, indem er diese beim Wort nahm und somit elegant-subversiv seinem Missmut Luft machte, ohne dass man ihm daraus hätte einen Strick drehen können: So betont er immer wieder, dass eine sorgfältige Übersetzung das Ansehen des Verlages stärke und damit auch im Interesse der DDR liege, die sich so nämlich durch ein qualitativ untadeliges Literaturprogramm vor den westdeutschen Verlagen auszeichnen könne, oder, erneut den höheren Zeitaufwand und die enstandenen Mehrkosten rechtfertigend:

Ich kann dazu nur sagen, was anlässlich einer kürzlichen Diskussion im Marmorsaal des hiesigen Hauses der Deutsch-Sowjetischen Freundschaft von Schriftstellem zum Ausdruck gebracht wurde: Ein Kunstwerk wächst nicht über Nacht!22

Er versucht auch gelegentlich, den sozialistischen Kulturbetrieb, und die Aufbau-Mitarbeiter sieht er letztlich als dessen Repräsentanten -, mit ihren eigenen Waffen zu schlagen, was den ideologischen Diskurs angeht. So heizt er das vom Kalten Krieg inspirierte Konkurrenzdenken zwischen west- und ostdeutschen Verlagen an, indem er darauf verweist, dass er mit einer gewissenhaften und philologisch einwandfreien Übersetzung seinen

21 Brief von Günter Caspar an die Verlagsleitung vom 3. Oktober 5? Jahreszahl

nicht lesbar); Archiv Aufbau-Verlag, SBB IIIA Dep38 A0384 0186 r [186 von 267].
${ }^{22}$ Ebenda. 
Beitrag dazu leiste, dass die DDR-Publikationen im Vergleich mit bundesdeutschen Ausgaben nicht nur bestehen, sondern sogar als Sieger hervorgehen können.

Es geht im übrigen, wenn man ein wenig zwischen den Zeilen liest, aus den Verlagsunterlagen hervor, dass die Verlagsleitung ihre Mitarbeiter mit all ihren Ecken und Kanten, aber auch ihren Vorzügen, gut kannte, so auch Krügel und Antkowiak. Mit Krügel wollte sie zusammenarbeiten, mit Antkowiak, der sich schon in anderen Verlagen als unangenehmer und allen progressiven Bestrebungen entgegentretender Mitarbeiter hervorgetan hatte, musste sie wohl auskommen, auch wenn man damals sicherlich nichts von seiner IM-Tätigkeit wusste. So schreibt Cheflektor Caspar (ab 1956 Cheflektor und ab 1963 Lektoratsleiter) in einem abschließenden Kommentar zu diversen Gutachten zur Ausgabe von ausgewählten Werken Eças, Antkowiaks Vorschlag erscheine ihm seriös, "wenn man auch bei ihm, wie immer, Einschränkungen machen mu $\beta^{\prime 2}{ }^{23}$ Über Krügel wiederum, in seinen Augen ein "guter und zuverlässiger Übersetzer" ${ }^{24}$, heißt es in einem Schreiben vom 30.5 .60 von dem Lektor und späteren Verlagsleiter Fritz-Georg Voigt ${ }^{25}$ an den Kaderleiter der Hochschule für Ökonomie in Hinblick auf eine eventuelle Lehrtätigkeit Krügels an der Hochschule, vermutlich als Portugiesischdozent:

Was die sozialistische Erziehung der Studenten betrifft, so darf man wohl von ihm nicht allzuviel erwarten, schon allein aus dem Grunde, weil er sich jahrelang vor allem in seine Übersetzungsarbeit vergraben hat. ${ }^{26}$

Wenn man berücksichtigt, wie wenig unverblühmt sich Krügel wiederholt dem DDR-Regime gegenüber geäußert hat, so lässt diese Formulierung nur die Schlussfolgerung zu, dass Voigt ihn mit diesem Hinweis hat schützen wollen.

\footnotetext{
${ }^{23}$ Archiv Aufbau-Verlag, SBB IIIA Dep38 A0197a 0078 r.

${ }^{24}$ Ebenda.

${ }^{25}$ Fritz-Georg Voigt, seit 1952 Lektor im Aufbau Verlag, übernahm 1966 die Verlagsleitung.

${ }^{26}$ Archiv Aufbau-Verlag, SSB IIIA Dep38 A0384 0048 [49 von 267].
} 
Aus den in diesem Beitrag zitierten Unterlagen geht hervor, dass der Aufbau Verlag größten Wert auf philologisch sorgfältig angefertigte Ubersetzungen legte. Diese waren mit ausführlichen Anmerkungen, Fußnoten und wissenschaftlich fundierten Vorworten versehen (im Falle Eças durch den renommierten linken portugiesischen Literaturwissenschaftler Óscar Lopes). Zur Klärung von Zweifeln und Begrifflichkeiten wandte man sich an sogenannte Außenmitarbeiter vor Ort im Ausland. Was konkret den Übersetzer Krügel angeht, so wird desweiteren deutlich, dass den Verantwortlichen des Aufbau Verlags sehr wohl bewusst war, dass Krügel in keiner Weise ein vorzeigbarer Repräsentant der Ideologie des Arbeiter- und Bauernstaats war. Da ihnen jedoch an der seiner sorgfältigen Übersetzungsarbeit gelegen war, trat man immer wieder mit Aufträgen an ihn heran und unterstützte ihn, so weit es ging. Hier zeigt sich auch ein Moment von Unabhängigkeit vom politischen Druck, das sich der Aufbau-Verlag bewahren konnte.

\section{BIBLIOGRAPHIE}

BARCK, Simone, Antifa-Geschichte(n). Eine literarische Spurensuche in der DDR der 1950er und 1960er Jahre, Köln, Böhlau Verlag, 2003.

CAL, Emesto Guerra da, Linguagem e Estilo de Eça de Queirós, Lissabon, Editorial Aster, 1954.

KiRSTEN, Jens, Lateinamerikanische Literatur in der DDR. Publikations- und Wirkungsgeschichte, Berlin, Christoph Links Verlag, 2004.

REIS, Carlos (ed.), A Obra Queirosiana no espaço da Língua Alemã. Santa Cruz do Douro, Fundação Eça de Queirós, 2011.

DeLILLE, Karl Heinz, „Deutsche Übersetzer und Übersetzerinnen des Eça de Queiros: ein Überblick", in: Suplementos de Lusorama, 2. Reihe, Band XVIII, Frankfurt, 2006, págs. 59-69. 